\section{Successful Use of Adalimumab for Treating Rheumatoid Arthritis with Autoimmune Sensorineural Hearing Loss: Two Birds with One Stone}

\section{To the Editor:}

There is considerable evidence to suggest that hearing and vestibular function can be influenced by autoimmune processes. In 1979, McCabe $^{1}$ reported 18 cases of patients with progressive bilateral hearing loss who responded to corticosteroids and/or cyclophosphamide. An autoimmune mechanism was suggested and he called this entity "autoimmune sensorineural hearing loss" (ASHL). A number of systemic autoimmune disorders include hearing loss and vertigo among their constellation of symptoms. Although evidence for a specific autoimmune etiology mediated by an immune response directed solely at the inner ear is elusive, it appears that autoimmune damage can also exist as an entity confined to the labyrinth. Antigenic targets of autoimmunity within the labyrinth seem to be diverse. Partly because of this, the condition is difficult to diagnose ${ }^{2}$. Various tests can be used to detect specific antibodies directed against antigens, such as $58 \mathrm{kDa}$ protein ${ }^{3}, 68 \mathrm{kDa}$ protein ${ }^{2}$, and $\mathrm{P} 0$ protein $^{4}$. The reactivity of patient sera against tissue sections of the inner ear has led to convincing labeling of sensorineural structures in some cases, but remains primarily a research tool ${ }^{5,6}$. Diagnosis of ASHL is therefore based on clinical findings and on responsiveness to steroid therapy. Immune-mediated inner ear disease includes clinical conditions associated with rapidly progressive unilateral or bilateral forms of sensorineural hearing loss. A systemic autoimmune disorder may be present in less than one-third of cases ${ }^{7}$. ASHL has been reported to occur in association with autoimmune diseases such as rheumatoid arthritis, systemic lupus erythematosus, Sjögren's syndrome, polyarteritis nodosa, relapsing polychondritis, Cogan's disease, and Crohn's disease ${ }^{8}$.

Autoimmune disorders of the inner ear are of special interest as they are among the few forms of hearing loss amenable to medical treatment. We describe a patient with hearing loss due to an autoimmune disease associated with rheumatoid arthritis (RA) whose hearing was improved by tumor necrosis factor- $\alpha$ (TNF- $\alpha$ ) blockade with adalimumab (Humira; Abbott Laboratories).

A 30-year-old woman had a 15-year history of RA, with numerous complications and frequent relapses. In 2009 she presented to our rheumatology clinic complaining of bilateral hearing loss. She had no history of vertigo or ocular problems. Otoscopic examination was normal. Neurological examination was unremarkable and in particular there were no cere-

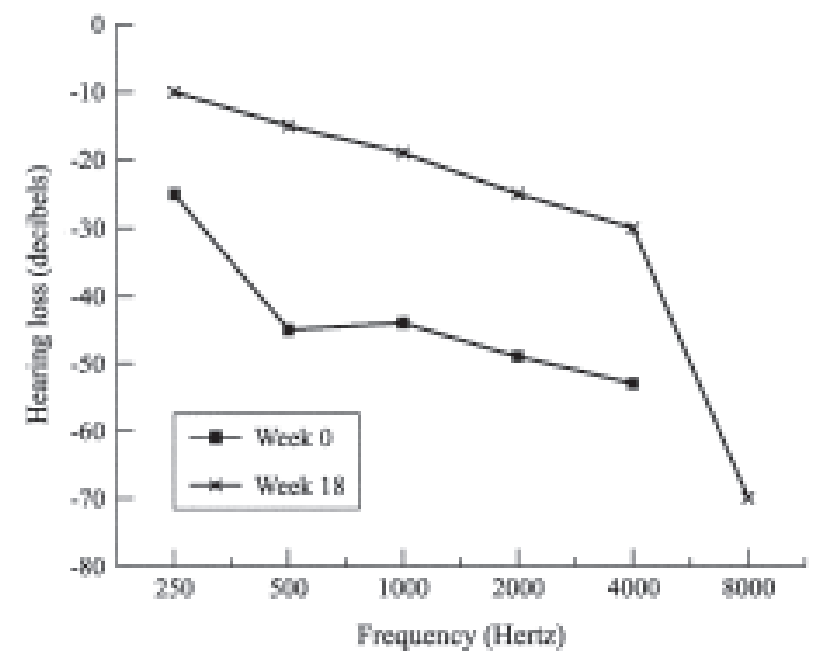

Figure 1. Hearing improvement in response to adalimumab. Serial audiograms from both ears demonstrating $15-30 \mathrm{~dB}$ improvement in the $250-4000 \mathrm{~Hz}$ range within the first 12 weeks of treatment. bellar or vestibular findings. Magnetic resonance imaging examination was normal. The audiogram (Figure 1) showed a down-sloping symmetric sensorineural hearing loss (ski-slope loss) with hearing loss predominantly for the high frequencies. Air and bone conduction were similarly impaired. Speech discrimination scores were determined as $90 \%$ for both ears. Diagnosis of ASHL was based on the clinical findings (i.e., bilateral rapidly progressive sensorineural hearing loss) and the presence of a systemic autoimmune disorder (RA). Prednisone $1 \mathrm{mg} / \mathrm{kg}$ per day and transtympanic injection of prednisone were used during 4 weeks, with no clinical improvement. Followup audiometry showed no audiometric improvement. Anti-TNF- $\alpha$ adalimumab was started for RA, as well as maintenance methotrexate therapy. Adalimumab $40 \mathrm{mg} /$ week was given subcutaneously. The patient noted a rapid improvement of her hearing. Serial audiometry confirmed a $15-30 \mathrm{~dB}$ improvement in the $250-4000 \mathrm{~Hz}$ range in each ear after 12 weeks. After 3 months of therapy she achieved remission of RA according to the 28-joint Disease Activity Score (DAS28).

To date the use of adalimumab for RA with ASHL has not been reported. Our patient with RA and ASHL did not respond to steroids, but was successfully treated with adalimumab. One of the most prominent proinflammatory mediators in the pathogenesis of RA is TNF- $\alpha$. TNF- $\alpha$-blocking agents bind with TNF- $\alpha$ molecules, neutralizing its biological activity, resulting in reduction of joint inflammation. Treatment strategies for ASHL are usually controversial; the pathogenesis of ASHL remains unclear, but antibodies directed to the inner ear and/or cellular effectors have been proposed. Multiple potentional mechanisms have been identified that can result in immune-mediated ASHL pathology ${ }^{9}$. In cases of ASHL, the inner ear inflammation progresses to severe, irreversible damage within a few months of onset (and often much more quickly). At the same time, responsiveness to steroid is high, and hearing loss may be reversible with prompt treatment. ASHL in association with some autoimmune diseases such as RA has a lower response to steroids, as in our patient ${ }^{10}$. TNF- $\alpha$ blockade by specific antibodies may offer an additional treatment option for patients with autoimmune hearing loss.

Our case demonstrates the efficacy of adalimumab in the management of RA with ASHL that did not respond to steroids. Further studies into the use of adalimumab in patients with RA and ASHL are warranted.

JADRANKA MOROVIC VERGLES, MD, PhD, Professor, Department of Rheumatology and Clinical Immunology, Dubrava University Hospital; MISLAV RADIC, MD, Department of Rheumatology, University Hospital Split, Split; JELENA KOVACIC, MD, Department of

Otorhinolaryngology; LEA SALAMON, MD, Department of

Rheumatology and Clinical Immunology, Dubrava University Hospital, Zagreb, Croatia.

\section{REFERENCES}

1. McCabe BF. Autoimmune sensorineural hearing loss. Ann Otol Rhinol Laryngol 1979;88:585-9.

2. Ryan AF, Keithley EM, Harris JP. Autoimmune inner ear disorders. Curr Opin Neurol 2001;14:35-40.

3. Boulassel MR, Tomasi JP, Deggouj N, Gersdorff M. COCH5B2 is a target antigen of anti-inner ear antibodies in autoimmune inner ear diseases. Otol Neurotol 2001;22:614-8.

4. Cao MY, Dupriez VJ, Rider MH, Deggouj N, Gersdorff MC, Rousseau GG, et al. Myelin protein Po as a potential autoantigen in autoimmune inner ear disease. FASEB J 1996;10:1635-40.

5. Soliman AM, Zanetti F. Improvements of a method for testing autoantibodies in sensorineural hearing loss. Adv Otorhinolaryngol 1988;39:13-7.

6. Soliman AM. Optimizing immunofluorescence for the testing of autoantibodies in inner ear disorders. Arch Otorhinolaryngol 1988;245:28-35.

7. Welling DB. Clinical evaluation and treatment of immune-mediated inner ear disease. Ear Nose Throat J 1996;75:301-5.

8. Froehlich F, Fried M, Gonvers JJ, Saraga E, Thorens J, Pecoud A. 
Association of Crohn's disease and Cogan's syndrome. Dig Dis Sci 1994;39:1134-7.

9. Yehudai D, Shoenfeld Y, Toubi E. The autoimmune characteristics of progressive or sudden sensorineural hearing loss. Autoimmunity 2006;39:153-8.

10. Dayal VS, Ellman M, Sweiss N. Autoimmune inner ear disease: clinical and laboratory findings and treatment outcome.

J Otolaryngol Head Neck Surg 2008;37:591-6.

J Rheumatol 2010;37:5; doi:10.3899/jrheum.091342 